\title{
Source of Reaction-Diffusion Coupling in Confined Systems due to Temperature Inhomogeneities
}

\author{
A. V. Anil Kumar, ${ }^{1}$ S. Yashonath, ${ }^{1,2}$ and G. Ananthakrishna ${ }^{3,2}$ \\ ${ }^{1}$ Solid State and Structural and Chemistry Unit, Indian Institute of Science, Bangalore, India 560012 \\ ${ }^{2}$ Center for Condensed Matter Theory, Indian Institute of Science, Bangalore, India 560012 \\ ${ }^{3}$ Materials Research Center, Indian Institute of Science, Bangalore, India 560012
}

\begin{abstract}
Diffusion is often accompanied by a reaction or sorption which can induce temperature inhomogeneities. Monte Carlo simulations of Lennard-Jones atoms in zeolite $\mathrm{NaCaA}$ are reported with a hot zone presumed to be created by a reaction. Our simulations show that localized hot regions can alter both the kinetic and transport properties. Further, enhancement of the diffusion constant is greater for larger barrier height, a surprising result of considerable significance to many chemical and biological processes. We find an unanticipated coupling between reaction and diffusion due to the presence of hot zone in addition to that which normally exists via concentration.
\end{abstract}

Diffusion within porous materials or confined geometry is still poorly understood $[1,2]$ despite increased attention in recent times [3,4]. Life sciences has a number of instances which relate to diffusion within confined regions - for example, ion diffusion across membranes and approach of a substrate towards an active site of an enzyme [5]. Hydrocarbon separation and catalysis within zeolites provide instances of processes in chemistry [6]. Problems involving fluid flow and excitonic transport through a porous medium are examples from physical sciences [2]. The richness of the subject partly arises from the geometry of confined systems (the fractal nature of the pores, for instance). Further, while nonuniformity of concentration has been dealt with in great detail, that of temperature has received little attention. In particular, when temperature is inhomogeneous, the very definition of diffusion as being an activated process needs a generalization. Such nonuniformity in temperature arises routinely in biological, chemical, and physical systems for a variety of reasons. Here, we discuss issues relating to the possible sources of such hot spots and their influence on transport properties in the context of zeolites.

Zeolites are porous solids with pore sizes comparable to molecular dimensions [7]. Because of its rich and diverse catalytic as well as molecular sieve properties [8] it has attracted much attention. The existence of specific catalytic and physisorption sites coupled with their poor thermal conductivity could lead to local hot regions [8]. (Typically in $10 \mathrm{ps}$, the hot region decays less than a few percent.) This may affect both kinetic and diffusion properties. Such a situation can arise in many biosystems as well. For instance, plasma membrane protein-encoding $m$-RNA IST2 is shown to have high asymmetry in concentration between the mother cell and the bud [9]. One possible way of maintaining such an asymmetry against the concentration gradient is through localized hot or cold regions. In spite of the importance of such reaction induced hot spot and its influence on the diffusion of the species, this problem has not been addressed so far.

Here, we study the effect of inhomogeneous temperature presumed to be created by a "reaction," on the equilibration rate and self-diffusion coefficient of guest molecules in zeolites. Monte Carlo simulations on simple argon atoms in zeolite $A$ are reported here. Our results show that selfdiffusion coefficient $D$ is increased substantially due to the presence of a hot zone. More significantly, at a conceptual level our analysis shows that local changes in temperature resulting from reactions can induce additional coupling between reaction and diffusion.

Landauer [10], in a seminal paper, addressed the effect of a nonuniform temperature bath on the relative occupation of competing local energy minima. For the case of a bistable potential $U(x)$ (the curve $A B C D$ in Fig. 1), he showed that the presence of a localized heating in a region (say $B C$ ) lying between the lower energy minimum $A$ and the potential barrier maximum $C$ can raise the relative population of the higher energy minimum $D$ over that given by the Boltzmann factor $\exp \left(-\Delta E / k_{B} T\right)$. This has come to be known as the "blowtorch" effect [10]. Since this effect is rather counterintuitive, following Landauer, we convey the basic idea. Consider the motion of an overdamped particle in this potential (curve $A B C D$ in Fig. 1) subject to a uniform temperature $T_{0}$ along the

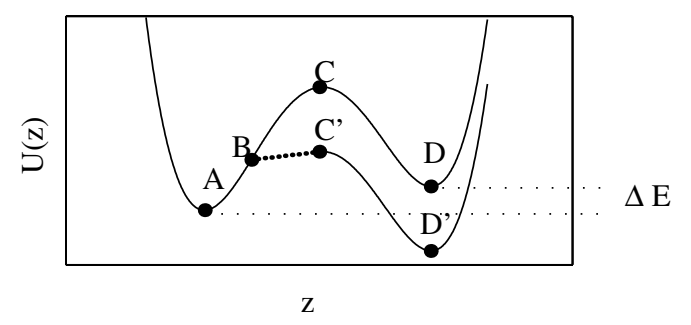

FIG. 1. The effect of a hot zone at $B C$ in the potential $A B C D$ is to lower $D$ to $D^{\prime}$. 
coordinate. Then, the probability of finding a particle at $x$ is $P(x) \sim \exp \left[-U(x) / k_{B} T_{0}\right]$. Then the probability at $A$ is higher than that at $D$. If the temperature in $B C$ is raised to $T_{b}$, then $P(x) \sim \exp \left[-U(x) / k_{B} T_{b}\right]$ in $B C$, which is much smaller than $P(x)$ with $T=T_{0}$ and hence $\log [P(x)]$ is flat in $B C$. Since $-\log P(x)$ can be considered as "potential" $U(x) / k_{B} T$, raising the temperature to $T_{b}$ in BC is equivalent to modifying the potential to flatter curve $B C^{\prime}$ (Fig. 1). Since $P(x)$ is unaffected in other regions, the curve outside $B C$ will be the same except that the curve $C D$ would start at $C^{\prime}$ and end at $D^{\prime}$ such that $U\left(x_{C}\right)-U\left(x_{D}\right)=U\left(x_{C^{\prime}}\right)-U\left(x_{D^{\prime}}\right)$. Thus, the point $D$ is brought down relative to $A$. Consequently, $P\left(x_{D}\right)$ is higher than at the lower minimum $x_{A}$.

A decade later an appropriate diffusion equation for a nonuniform temperature profile was derived by van Kampen [11], followed by additional work from Landauer himself [12]. Recently, Bekele et al. [13] have shown that the escape rate is enhanced due to the presence of a hot zone. This implies that the rate of surmounting the barrier in zeolite will be increased by the presence of a hot zone and a consequent increase in diffusion constant.

The physical system we simulate consists of $\mathrm{NaCaA}$ zeolite with large $(\approx 11.5 \AA$ diam) cages (the supercages) interconnected by shared narrow eight-ring windows $(\approx 4.5 \AA$ diam $)$. The potential energy landscape has a maximum near the eight-ring window and a minimum located deep within the supercage. A species arriving at a heterogeneous reaction site, assumed to be located between the window and the center of the cage (Fig. 2) releases a heat $q$ creating a local hot zone. Consequently, the molecule surmounts the barrier more easily. Other molecules behind it also cross the barrier with relative ease due to the hot zone. Here, we mimic the reaction by its principal effect - the presence of a hot zone - by introducing it in between the potential maximum and minimum. In the case of physisorption we assume that

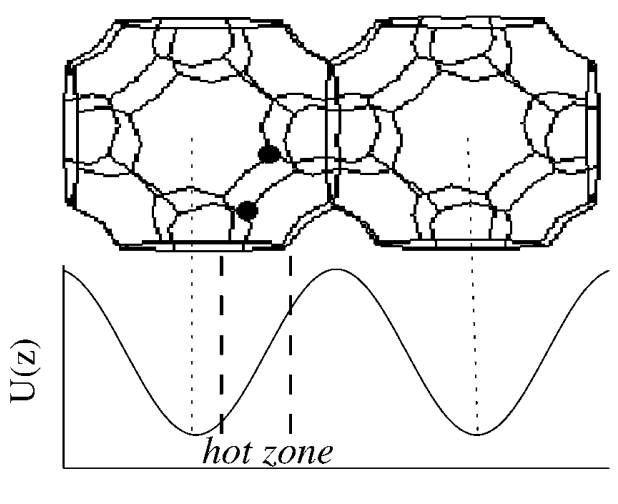

Z

FIG. 2. Two cages of zeolite NaCaA. - show reaction sites. The saddle point is at the window. A schematic one-dimensional potential along the $z$ direction is shown below along with the induced hot zone. For set $A$ simulations the potential at extremes of $z$ goes to infinity (not shown). For set $B$, the potential shown above is periodic. the heat released due to sorption is taken up by another diffusing particle. The effect arising out of the occupied volume of the adsorbent is ignored as it is negligible (for methane-NaY, this is typically $2 \%$ of the cage volume).

We consider Lennard-Jones particles confined to $2 \times$ $2 \times 1$ unit cells of $\mathrm{NaCaA}$ zeolite. There are $2^{3}$ cages in each unit cell and $4 \times 4 \times 2$ cages in the $x, y$, and $z$ directions. The potential along the $z$ direction is a symmetric double well (Fig. 2). Since the rate determining step is the passage through the eight-ring window, the distance of the particle from this plane may be considered as the diffusion coordinate. Because of the lack of appropriate techniques for including nonuniformity in temperature (that is, in maintaining steady state excess temperature in a localized region) within the existing molecular dynamics algorithms, we use Metropolis Monte Carlo algorithm in the canonical ensemble where the total energy is

$$
\Phi=\sum_{g} \sum_{z} \phi_{g z}+\frac{1}{2} \sum_{g} \sum_{g} \phi_{g g}
$$

and $\quad \phi_{a b}=4 \epsilon_{a b}\left[\left(\sigma_{a b} / r\right)^{12}-\left(\sigma_{a b} / r\right)^{6}\right] ; a, b=g, h$ is the Lennard-Jones potential. Thus, the particle diffuses on the potential energy landscape of the zeolite. The potential parameters for the guest-guest and guest-host interactions are $\sigma_{g g}=2.73 \AA$ and $\epsilon_{g g}=$ $0.9977 \mathrm{~kJ} / \mathrm{mol} . \sigma_{\mathrm{O}-\mathrm{O}}=2.5447 \AA, \sigma_{\mathrm{Na}-\mathrm{Na}}=3.3694 \AA$, $\sigma_{\mathrm{Ca}-\mathrm{Ca}}=3.35 \AA, \quad \epsilon_{\mathrm{O}-\mathrm{O}}=l^{*} 1.28991 \mathrm{~kJ} / \mathrm{mol}, \quad \epsilon_{\mathrm{Na}-\mathrm{Na}}=$ $l^{*} 0.03924 \mathrm{~kJ} / \mathrm{mol}$, and $\epsilon_{\mathrm{Ca}-\mathrm{Ca}}=l^{*} 9.5451 \mathrm{~kJ} / \mathrm{mol}[14]$ where $l=2,4$. The cross terms are obtained from Lorentz-Berthelot combination rule. The cutoff distance was $12 \AA$.

Two different sets of simulations $A$ and $B$ are carried out. In set $A$, for the calculation of the escape rate, we impose periodic boundary conditions (PBC) along the $x$ and $y$ directions and a repulsive potential $\left(1 / r^{12}\right)$ at both ends along $z$ direction which enables a comparison with earlier work [13]. In set $B$, for the calculation of $D$ in three dimensions, $\mathrm{PBC}$ along all three directions is essential. We investigate the influence of degree of hotness defined by $s=\left(T_{b}-T_{0}\right) / T_{0}$ and the barrier height $U_{a}(\propto l \epsilon)$ on the equilibration rate and $D$. Here, $T_{0}$ and $T_{b}$ are the background and blow torch temperatures, respectively. $T_{0}$ is kept at $300 \mathrm{~K}$, and $T_{b}$ is varied.

Initially, all the 64 guest particles, at a concentration of 2 atoms/supercage are uniformly distributed in the four left cages located along the $z$ direction (see Fig. 1). It is well known that transport properties are determined by the time scales associated with the approach to the steady state. These are obtained by allowing the system to evolve towards the steady state in the presence of the hot zone. Let $n_{l}$ and $n_{r}$ be, respectively, the total number of particles in the cages to the left and the right of saddle point along the $z$ direction. Then, the decay rate is obtained by plotting the fraction of particles in the left cages as a function of time. A typical plot of $\ln \left[n_{l} /\left(n_{l}+n_{r}\right)\right]$ vs $t$ is shown in Fig. 3. The slope then gives the equilibration rate. The 


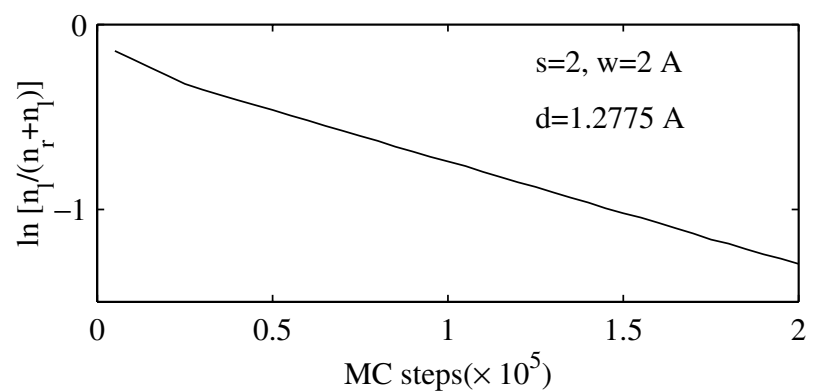

FIG. 3. A typical plot of $\ln \left[n_{l} /\left(n_{l}+n_{r}\right)\right]$ vs MC steps obtained from MC runs (set $A$ ).

curve shown in Fig. 3 will reach a plateau at much longer time scale.

We consider the influence of the hot zone on the rate of approach to the steady state as a function of $s$ when the hot zone of width $w=2 \AA$ is placed at a distance $d=1.2775 \AA$ from the window. Instead of the escape rate, we use the enhancement factor $f_{b}$, which is the ratio of the escape rates from the left cage with and without the hot zone. A plot of $f_{b}$, as a function of $s$, for two values of $l$ is shown in Fig. 4. The tendency to approach saturation is seen for both values of $U_{a}(\propto l \epsilon)$, even though it is less pronounced for the higher value $(l=4)$. More importantly, $f_{b}$ is a sensitive function of the barrier height [13] (Fig. 4). Thus, the enhancement in $f_{b}$ is greater when $U_{a}$ is larger which implies larger enhancement in $D$ for systems with higher energy barriers.

The significance of these results becomes apparent on examining a real system such as methane in faujasite. The energy at the physisorption site for methane in $\mathrm{NaY}$ zeolite (with $\mathrm{Si} / \mathrm{Al}=3.0$ ) is $-18 \mathrm{~kJ} / \mathrm{mol}$, the energy difference between a free methane and a physisorbed methane. However, the energy released is significantly lower $(\sim-6 \mathrm{~kJ} / \mathrm{mol})$ when it is already within the zeolite [15]. This can raise the temperature in the vicinity of the site.

We now consider the influence of a hot zone on diffusion through set $B$ simulations for which the starting configuration is the final configuration of set $A$. We have studied the influence of $s$, and the barrier height. The ratio of the diffusion constant $D_{h}$ with the hot spot to that without, $D_{0}$ is enhanced in each case (Table I). We note that since all other conditions of the simulation are identical in the two

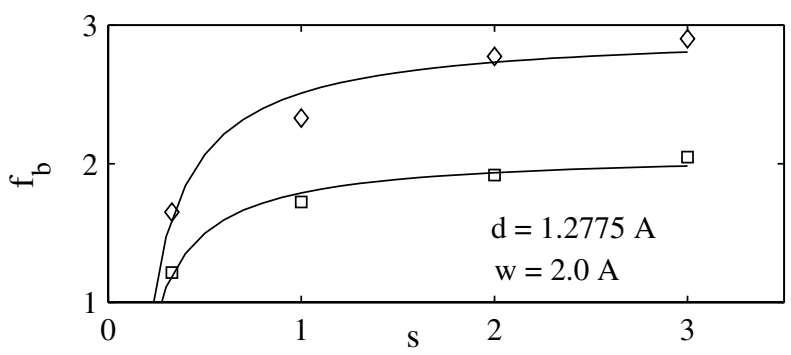

FIG. 4. A plot of the enhancement factor $f_{b}$ as a function of the degree of hotness $s=\left(T_{b}-T_{0}\right) / T_{0}$ for two different values of well depth $l \epsilon[l=2(\square), 4(\diamond)]$ from MC runs (set $A)$. situations, $D_{h} / D_{0}$ is independent of the basic length and time scales and also the details of the simulation such as the particle displacement (similarly for set $A$ ). Note that the larger the barrier height greater is the enhancement in $D$, a result that has considerable implication. These results are better understood on the basis of simple arguments to estimate $D_{h} / D_{0}$.

In the Kramers picture, the slowest time scale determining the approach to the steady state is identified with the escape rate. To estimate this, consider a one-dimensional symmetric double well potential with PBC. The rate equation for the number of particles to the left $\left(n_{l}\right)$ and right $\left(n_{r}\right)$ of the potential maximum, is $\dot{n}_{l}=-w_{l r} n_{l}+w_{r l} n_{r}$, where $w_{l r}$ and $w_{r l}$ are the escape rates from the left to the right well and vice versa, respectively. These are also the very time scales that determine the steady state through detailed balance condition: $\dot{n}_{l}=0$ or $w_{l r} n_{l}=w_{r l} n_{r}$. In the absence of hot spot, we have $w_{l r}=w_{r l}$. Then the mean escape rate from a well is $w_{0}=\left(w_{l r}+w_{r l}\right) / 2$. Then the diffusion constant in one dimension, $D_{0}=a^{2} w_{0} / 2$, where $a$ is the distance between the two minima. On introduction of a hot zone in the left well, $w_{l r}$ has been shown to increase considerably, while $w_{r l}$ increases only marginally [13], and to a good approximation $w_{r l} \approx w_{0}$. The mean escape rate is $w_{h}=\left(w_{l r}+w_{r l}\right) / 2$. Using this with $w_{l r}=w_{r l} n_{r} / n_{l}$, we get $D_{h}=a^{2} w_{h} / 2 \approx D_{0}(1+$ $\left.n_{r} / n_{l}\right) / 2$. Since, $n_{r} / n_{l}>1$ in the presence of a hot zone, it is evident that $D$ is enhanced. Note that this refers to a nonequilibrium inhomogeneous situation as $n_{r} / n_{l}$ refers to the steady state which can only be obtained numerically. Table I, shows the values of $D_{h} / D_{0}$ obtained from the mean square displacement through $\mathrm{MC}$ simulations for three sets of parameter values along with $D_{h} / D_{0}$ estimated from the above expression using the steady state values of $n_{r} / n_{l}$ from MC simulations. (Note $n_{r}$ and $n_{l}$ refer to the total number of particles to the left and right of the potential maxima between the two cages in the $z$ direction.) Clearly, the estimated values are close to $\mathrm{MC}$ values.

To facilitate comparison of these results with real systems, we have estimated the likely increase in temperature when hydrocarbons and other guest species are sorbed within zeolites such as $\mathrm{NaX}$. We have listed in Table II isosteric heat of sorption $\left(\Delta H_{\mathrm{ads}}\right)$ of some linear alkanes, $\mathrm{Xe}$, and water within faujasites. We have also listed the mean heat capacities $\left(C_{m}\right)$ of the guest-zeolite systems [7]. From these data, the maximum increase in $\Delta T$ can be estimated from $\Delta T=T_{b}-T_{0}=\Delta H_{\text {ads }} / C_{m}$ which is in the range 820 to $2028 \mathrm{~K}$ (Table II). Thus, the parameter $s$

TABLE I. $\quad D_{h} / D_{0}$ for different sets of parameters. Here $d=$ $1.2775 \AA$ and $w=2 \AA$ from MC runs (set $B)$.

\begin{tabular}{ccccc}
\hline \hline$s$ & $\begin{array}{c}l(\text { in } \\
\left.\epsilon=l * \epsilon_{g z}\right)\end{array}$ & $n_{r} / n_{l}$ & $\begin{array}{c}D_{h} / D_{0} \\
\text { (estimated) }\end{array}$ & $\begin{array}{c}D_{h} / D_{0} \\
(\mathrm{MC})\end{array}$ \\
\hline 2 & 2 & 2.0175 & 1.5088 & 1.4283 \\
3 & 2 & 3.5416 & 2.2703 & 2.1971 \\
2 & 4 & 2.6132 & 1.8066 & 1.6747 \\
\hline \hline
\end{tabular}


TABLE II. Expected rise in temperature and $s$ for typical guests when adsorbed in common zeolites estimated from heat of asorption $\Delta H_{\mathrm{ads}}$ and the mean heat capacity $C_{m}$ data.

\begin{tabular}{|c|c|c|c|c|c|c|}
\hline \multicolumn{2}{|c|}{ System } & \multirow{2}{*}{$\begin{array}{c}\Delta H_{\mathrm{vap}} \\
\mathrm{kJ} / \mathrm{mol}\end{array}$} & \multirow{2}{*}{$\begin{array}{l}\Delta H_{\mathrm{ads}}{ }^{\mathrm{a}} \\
\mathrm{kJ} / \mathrm{mol}\end{array}$} & \multirow{2}{*}{$\begin{array}{c}C_{m} \\
\mathrm{~J} / \mathrm{mol} . \mathrm{K}\end{array}$} & \multirow{2}{*}{$\begin{array}{c}T_{b}-T_{0} \\
\mathrm{~K}\end{array}$} & \multirow[b]{2}{*}{$s$} \\
\hline Guest & Zeolite & & & & & \\
\hline$n-\mathrm{C}_{4} \mathrm{H}_{10}$ & $\mathrm{Na}-X$ & 66 & 174 & 105 & $1689^{b}$ & 5.6 \\
\hline$n-\mathrm{C}_{7} \mathrm{H}_{16}$ & $\mathrm{Na}-X$ & 87 & 228 & 176 & $1809^{b}$ & 6.0 \\
\hline$n-\mathrm{C}_{7} \mathrm{H}_{16}$ & $\mathrm{Na}-X$ & 87 & 228 & 209 & $1090^{c}$ & 3.3 \\
\hline neo- $\mathrm{C}_{5} \mathrm{H}_{12}$ & $\mathrm{Na}-X$ & 54 & 130 & 129 & $1011^{b}$ & 3.3 \\
\hline iso- $\mathrm{C}_{8} \mathrm{H}_{18}$ & $\mathrm{Na}-X$ & 88 & 246 & 185 & $1329^{\mathrm{d}}$ & 4.0 \\
\hline $\mathrm{Xe}$ & $\mathrm{Na}-Y$ & $\ldots$ & 18 & 22 & $820^{\mathrm{e}}$ & 1.7 \\
\hline $\mathrm{H}_{2} \mathrm{O}$ & $\mathrm{Na}-X$ & . & 142 & 70 & $2028^{b}$ & 6.7 \\
\hline
\end{tabular}

${ }^{\mathrm{a} C a l c u l a t e d}$ from $\Delta H_{\mathrm{vap}}$ and the ratio of $\Delta H_{\mathrm{ads}}$ [7]. ${ }^{\mathrm{b}} T_{0}=$ $300 \mathrm{~K} ;{ }^{\mathrm{c}} T_{0}=333 \mathrm{~K} ;{ }^{\mathrm{d}} T_{0}=325 \mathrm{~K} ;{ }^{\mathrm{e}} T_{0}=473 \mathrm{~K}$.

varies from 1.7 to 6.7 for which $f_{b}$ can be as large as 3 for $s \sim 6$ and $l=2$. Since $f_{b}$ is determined by the very rate constants that lead to steady state, it also implies that the diffusion constant in the inhomogeneous medium can increase by a factor of 2, even for moderate values of $s$ as can be seen from Table I.

An interplay of reaction and diffusion is known to give rise to complex dynamics which can manifest in different ways [16]. The product profile in a reaction is controlled by the diffusion rate of the product species formed rather than the reaction rate as is the case with the formation of $p$ xylene in ZSM-5 [8]. Because of low diffusivity of ortho and meta isomers, they are not observed as products even though they are formed. The coupling between reaction and diffusion in such systems is via the concentration of the reactant and product species [17]. In contrast, in the present situation, the enhancement in $D$ is a direct consequence of inhomogeneous temperature. This study demonstrates that such a coupling between reaction and diffusion can arise not just due to concentration, but also due to the increase in local temperature, a fact that could not have been anticipated. These results also show how diffusion is increased in the presence of physisorption or chemisorption which are usually exothermic. $D$ may decrease if the reaction is endothermic.

The present analysis can provide an insight into a wellknown experimental observation where a warm adsorption front is seen to move rapidly during the adsorption of a gas into an evacuated zeolite [18]. As the initial molecules arrive at a physisorption site, heat is released which aids the molecules at the front to cross over the energy barrier and propel the gas forward. Zeolites are crystalline solids, and hence the active sites are located in a periodic manner. Thus, as the front moves further into the zeolite, hot zones are created successively providing a periodic driving force for diffusion which is in addition to that arising from the concentration gradient. This explains the rapid movement of the front.

Thus, influence of a reaction induced hot zone can affect diffusion in ways which will be important both from a fundamental as well as industrial perspective. Though our results are obtained in the context of zeolites, it is evident that they are of significance to many biological processes where concentration gradients are frequently accompanied by difference in temperature. These results also have implication to the separation of isotopes [19], the petrochemical industries, fast ion conducting battery materials, etc. We believe that in the foreseeable future, local inhomogeneities in temperature will be exploited in a number of ways to bring forth novel processes.

A. V.A.K. and S. Y. thank IUC, Indore for support, and financial support from DST, New Delhi (Project No. SP/S1/H-12/99).

[1] Complete issue of J. Chem. Soc. Faraday Trans. 87, 37 (1991).

[2] Molecular Dynamics in Restricted Geometries, edited by J. Klafter and J. M. Drake (Wiley, New York, 1989).

[3] S. P. Bates and R. A. van Santen, Adv. Catal. 42, 1 (1997).

[4] F. J. Keil, R. Krishna, and M-O. Coppens, Rev. Chem. Eng. 16, 71 (2000).

[5] A. L. Lehninger, D. L. Nelson, and M. M. Cox, Principles of Biochemistry (North Publications, New York, 1993).

[6] J. Karger and D. M. Ruthven, Diffusion in Zeolites and Other Microporous Solids (John Wiley and Sons, Inc., New York, 1992).

[7] R. M. Barrer, Zeolites and Clay Minerals as Sorbents and Molecular Sieves (Academic Press, London, 1978).

[8] J. M. Thomas, Philos. Trans. R. Soc. London A 333, 173 (1990); J. M. Thomas and W. M. Thomas, Principles and Applications of Heterogenous Catalysis (VMH, Weinheim, 1997).

[9] R. P. Brendza, L. R. Serbes, J. B. Duffy, and W. M. Saxton, Science 289, 2120 (2000).

[10] R. Landauer, Phys. Rev. A 12, 636 (1975).

[11] N. G. van Kampen, IBM J. Res. Dev. 32, 107 (1988).

[12] R. Landauer, J. Stat. Phys. 53, 233 (1988).

[13] M. Bekele, S. Rajesh, G. Ananthakrishna, and N. Kumar, Phys. Rev. E 59, 143 (1999).

[14] A. V. Kiselev and P. Q. Du, J. Chem. Soc. Faraday Trans. 2 77, 1-15 (1981).

[15] S. Yashonath, J. M. Thomas, A. K. Nowak, and A. K. Cheetham, Nature (London) 331, 601 (1988).

[16] K-J. Lee, W. D. McCormick, J. E. Pearson, and H. L. Swinney, Nature (London) 369, 215 (1994).

[17] P. Demontis, G. B. Suffritti, and A. Tilocca, J. Chem. Phys. 111, 5529 (1999).

[18] D. Basmadjian, K. D. Hu, and C-Y. Pan, Ind. Eng. Chem. Process Dev. 14, 328 (1975); 14, 340 (1975).

[19] H. Yamakawa et al., J. Nucl. Sci. Technol. 37, 710 (2000). 